\title{
Evaluation of an accelerated Ponseti protocol for the treatment of talipesequinovarus in Nigeria
}

\author{
G H. Ibraheem ${ }^{1}$, O O. Adegbehingbe ${ }^{2}$, O M. Babalola ${ }^{1}$, S B. Agaja ${ }^{1}$,
} B A. Ahmed ${ }^{1}$, A Olawepo ${ }^{1}$, M Medubi ${ }^{1}$

${ }^{1}$ Department of Orthopaedics, University of Ilorin Teaching Hospital, Ilorin, Nigeria

${ }^{2}$ Department of Orthopaedic Surgery \& Traumatology, Obafemi Awolowo University, lle-Ife, Nigeria

Correspondence to:Gbadebo H. Ibraheem, Email:ghibraheem@yahoo.com https://dx.doi.org/10.4314/ecajs.v22i1.4

Background: The commonest congenital musculoskeletal deformity worldwide is talipes equino varus (CTEV). Of the various treatment methods available, the most acceptable treatment currently for CTEV worldwide is serial manipulation and casting using the Ponseti technique. The Ponseti technique consists of weekly manipulation and casting for an average of six to eight weeks followed by bracing of the feet till school age. Some studies have shown evidence that more frequent casts may have similar outcomes with weekly casts using the Ponseti method. This study compared the efficacy of management of idiopathic CTEV using an accelerated protocol of twice weekly casts, with the standard weekly Ponseti protocol.

Methods: The study was a randomized case control study with a group of patients undergoing an accelerated casting protocol involving twice weekly casts compared with another group who went through the normal weekly Ponseti protocol.

Results: A total of $\mathbf{4 5}$ feet in $\mathbf{2 8}$ patients were studied. The study showed a significantly shorter treatment period in the accelerated protocol (39 days) compared with the standard protocol (52 days); with no significant increase in the number of casts needed for treatment.

Conclusions: A twice weekly Ponseti casting protocol enables completion of the casting period earlier than the standard Ponseti method.

Key words: Talipes equinovarus; Ponseti treatment; Accelerated Ponseti Protocol; Serial Casting

\section{Introduction}

Clubfoot refers to a group of disorders where the leg, ankle and foot are shaped like a hockey stick. It is a complex developmental anomaly of which majority of cases are congenital with a worldwide incidence of 1-6.8/1000 live births ${ }^{1}$. While a variety of distinct disorders fall under the description of 'clubfoot', the commonest type is congenital talipesequiovarus (CTEV). The CTEV deformity consists of equinus at the ankle, varus of the hindfoot, forefoot adduction and cavus ${ }^{2}$. Clubfoot is regarded as the most common and 


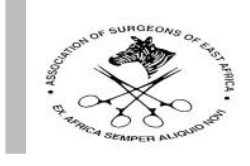

potentially debilitating congenital musculoskeletal deformity worldwide. It has been identified in the mummified remains of the Egyptian boy king Tutankhamen as well as in several other individuals in history as well as in contemporary times ${ }^{3}$.

Clubfoot is regarded as a developmental anomaly. A normally developing foot turns into a clubfoot during the second trimester of pregnancy. Ponseti described the clubfoot deformity as occuring mostly in the tarsus ${ }^{4}$. The tarsal bones, which are mostly made of cartilage, are in the most extreme positions of flexion, adduction, and inversion at birth. The talus is in severe plantar flexion, its neck is medially and plantarly deflected, and its head is wedge-shaped. The navicular is severely medially displaced, close to the medial malleolus, and articulates with the medial surface of the head of the talus. In addition, the calcaneus is adducted and inverted under the talus. The ligaments of the posterior and medial aspect of the ankle and tarsal joints are very thick and taut, thereby severely restraining the foot in equinus and the navicular and calcaneus in adduction and inversion. The soft tissues around the leg, foot and ankle are also abnormal in clubfoot. Excessive collagen synthesis in the ligaments, tendons, and muscles may persist until the child is 3 or 4 years of age and might be a cause of relapses ${ }^{4}$. Under the microscope, the bundles of collagen fibers display a wavy appearance known as crimp. This crimp allows the ligaments to be stretched. Gentle stretching of the ligaments in the infant causes no harm. The crimp reappears a few days later, allowing for further stretching. That is why manual correction of the deformity is feasible ${ }^{4}$.

A variety of classification systems based on clinical examination have been devised, the most common being the Pirani system which has been shown to have good interobserver reliability and reproducibility ${ }^{5,6}$. Children born with CTEV face a lifetime of pain in the feet and inability to wear normal shoes with the associated social stigmatization that comes from living with the deformity. The goal of treatment of clubfoot is to achieve a functional, pain-free, plantigrade foot with good mobility which does not require the patient to wear modified shoes? ${ }^{7}$.

Treatment of CTEV has evolved over the last century from exclusively non-operative management, to mainly surgical correction of the deformity and back to mainly nonoperative management. Historically, various methods of splinting, binding and casting have been explored over the many centuries during which clubfoot treatment has been recorded $^{8}$. These efforts largely gave way to surgical treatment of the deformity in the early 20th century via various approaches, the most popular of which was the posteromedial release ${ }^{9,10,11}$. These extensive posteromedial soft tissue releases were common before 1948, but often resulted in stiffness and recurrence ${ }^{12}$. Joseph Hiram Kite introduced the principles of non-operative management of clubfoot with his method of serial manipulation and casting. Kite reported a success rate of $90 \%$ in patients whose treatment was started before one year of age with a cast duration of 26-49 weeks ${ }^{13,14}$. Other surgeons were however unable to produce similar results with Kite's method, achieving only $20-50 \%$ success $^{15}$. In 1948, Ignatio V. Ponseti at the University of lowa, U.S.A, introduced his method of clubfoot treatment. He published the first results from patients he managed between 1948 and 1956 showing good results in $71 \%$ of his patients ${ }^{16}$. Acceptance of the technique was however 
slow in the mainstream orthopaedic community until the results of long term follow up for his patients over a range of $10-27$ years, showed that $90 \%$ of his patients were satisfied with their treatment outcomes ${ }^{12}$. Even longer follow-up results by Cooper and Dietz showed $78 \%$ excellent or good outcomes after a 30 year follow up ${ }^{17}$. The good long term results from Ponseti's method encouraged its adoption in many centres and several other surgeons were able to demonstrate high success rates using the Ponseti technique ${ }^{17,18,19,20}$. In the last decade, it has been established that of the various surgical and non-operative treatment methods available, the most acceptable treatment currently for CTEV worldwide is serial manipulation and casting using the Ponseti method ${ }^{21,22}$.

The Standard Ponseti clubfoot protocol traditionally consists of weekly manipulation and casting following a definite sequence to correct the deformity ${ }^{23}$. Immediately after removal of the final cast, the feet are placed in an abduction brace which the child wears throughout the day for the first three months. The bracing duration is then reduced gradually until night-time bracing alone is done till the child is about 4 years old. Strict observance of the bracing protocol is essential to prevent relapse or recurrence.

The relative cheap cost of the casting procedure makes the treatment process particularly relevant to low income communities where a larger percentage of the CTEV patients live. Because many patients with the disease live far away from centres with the expertise for treatment, some parents are forced to relocate to these towns or cities for the duration of the manipulation and casting process. Other parents and care-givers have also desired a shorter casting period to reduce the difficulties faced in handling the casts. These difficulties have prompted investigators to consider various accelerated casting protocols to achieve a more rapid correction of the deformity. It was first revealed that casts changed every five days, instead of the originally prescribed seven days, gave the same results - potentially saving ten to 12 days in the initial casting phase ${ }^{24}$. Some other studies have also shown evidence that more frequent casts may have similar outcomes with weekly casts using the Ponseti method ${ }^{25,26}$.

The study was aimed at comparing the efficacy of management of idiopathic CTEV using the standard Ponseti protocol on one hand and an accelerated Ponseti protocol involving twice weekly manipulation and casting on the other hand.

\section{Patients and Methods}

The study was a case control study whose subjects were infants in the first three months of life who presented with idiopathic CTEV. Ethical approval for the study was obtained from the Ethics and Research Committee of the hospital. Infants who presented with CTEV were divided to two treatment groups after giving consent for inclusion into the study. Patients were randomly assigned to either group by alternatively allocating successive patients to the control and test group in that order according to their chronological hospital numbers. Randomisation was done at the patient level with both feet being treated by the same method in cases of bilateral CTEV. 
The Standard Ponseti Protocol (SPP) was taken as the control group (group A), while the Accelerated Ponseti Protocol (APP) was the test group (group B). Group A was managed with the standard Ponseti protocol which consists of serial manipulation and casting which was done once weekly on Thursdays. The serial manipulation was done according to the technique described by Prof I. Ponseti with groin to toe casts applied after each manipulation until correction was achieved ${ }^{23}$.

The test group (group B) was managed with an accelerated protocol consisting of identical serial manipulation and casting which was however done twice weekly (on Mondays and Thursdays) until full correction was achieved.

\section{Treatment protocol ${ }^{23}$ :}

Before each cast is applied, the foot is manipulated. The first manipulation is aimed at correcting the cavus (high medial arch) by aligning the forefoot with the midfoot. Subsequently, the head of the talus is stabilised with the thumb of one hand while the forefoot is abducted with the talus serving as the fulcrum. The heel is not touched to allow the calcaneus to abduct with the foot. A light padding is applied on the limb from the toes to the groin. The foot is held in the maximum corrected position while a cast is applied from the toes to the knee. The cast is then molded, while maintaining correction. After moulding, the cast is then extended proximally to just below the groin. The casts are removed in the clinic at every visit and manipulation and casting is repeated weekly until all aspects of the deformity are corrected except the equinus. If there is a residual equinus deformity after other deformities are corrected, a percutaneous Achilles tenotomy is done in the clinic. The Achilles tenotomy involves severing the Achilles tendon about $1.5 \mathrm{~cm}$ above its insertion into the calcaneus. A final cast is applied with the foot in hyperabduction and the ankle in dorsiflexion. The final cast remains in place for three weeks to allow healing of the Achilles tendon in the elongated state.

Both groups were managed by the same investigator who is a Ponseti clubfoot practitioner of 5 years' experience. The severity of the deformity in all feet was scored using the Pirani scoring system at each presentation. The end point was determined as the day the last cast was removed after tenotomy had been performed (cavus, forefoot adduction, hind foot varus and equinus deformities are all corrected). This gave a plantigrade foot with Pirani score of 0 (foot abduction of $60^{\circ}$ and dorsiflexion of $15^{\circ}$ ).

Children who had open wounds or other soft tissue injuries on the legs or feet that prevented application of the casts and those who had had prior treatment before presentation were excluded from the study.

The data collected was analyzed using the IBM ${ }^{\circ}$ SPSS version $20.0^{\circ}$. Mean Pirani scores at the beginning of the treatment, and the number of days from application of the first cast to removal of the last cast was compared in both groups. The number of casts applied in both groups were also compared as well as the direct costs incurred for the casting and tenotomy in all patients. 
Results

A total of 45 feet in 28 patients were studied. 23 feet in 14 patients were managed with the standard Ponseti protocol (Control group) while 22 feet in 14 patients were managed with the accelerated protocol (Test group). The patients were not matched for age or sex. The patients' ages ranged from one to 90 days old at commencement of treatment with a mean age of 34.4 days in the SPP group and 56.9 days in the APP group. Male to female ratio was 2.5:1 in the SPP group and 1:1 in the APP group as shown in figure 1.

A total of $64 \%$ of the patients in the SPP group had bilateral CTEV, while only $57 \%$ of the patients in the APP group had bilateral CTEV as shown in figure 2. There was no significant difference in the Pirani scores at presentation between the SPP group (mean 5.0) and the APP group (mean 4.8) $(p=0.327)$.

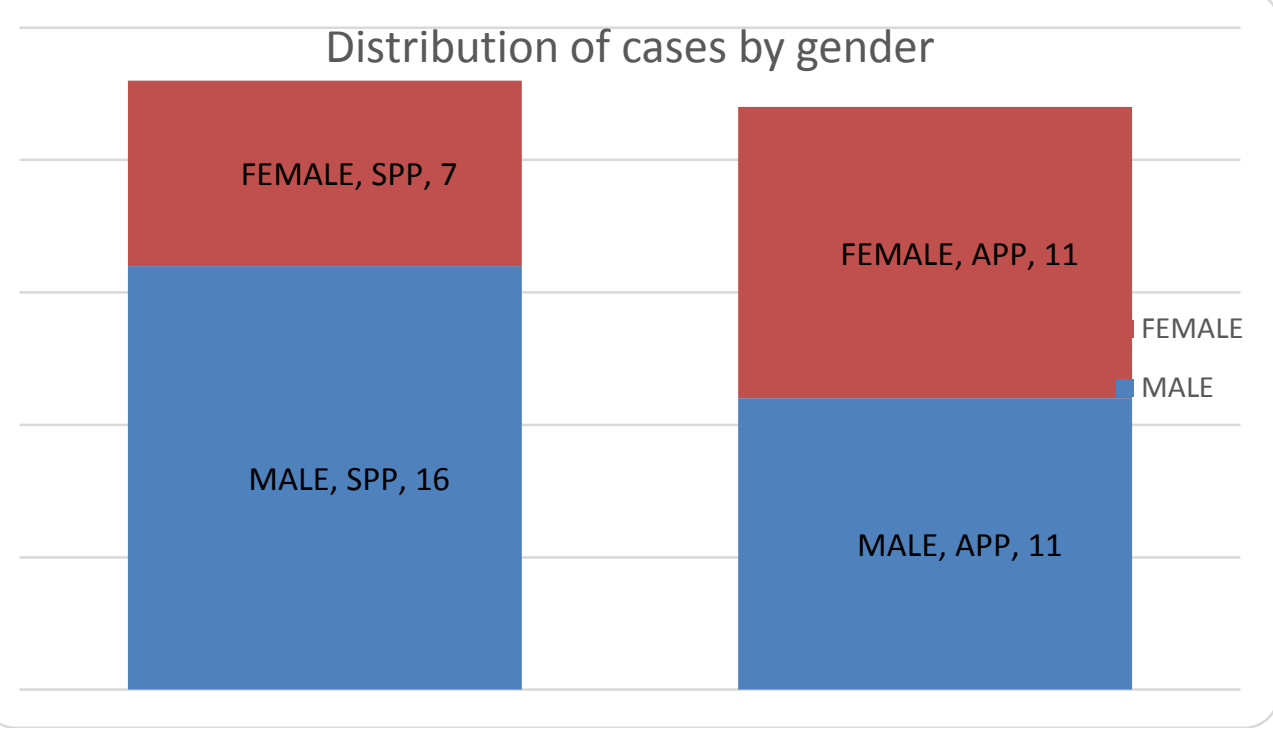

Figure 1. Distribution of cases by gender

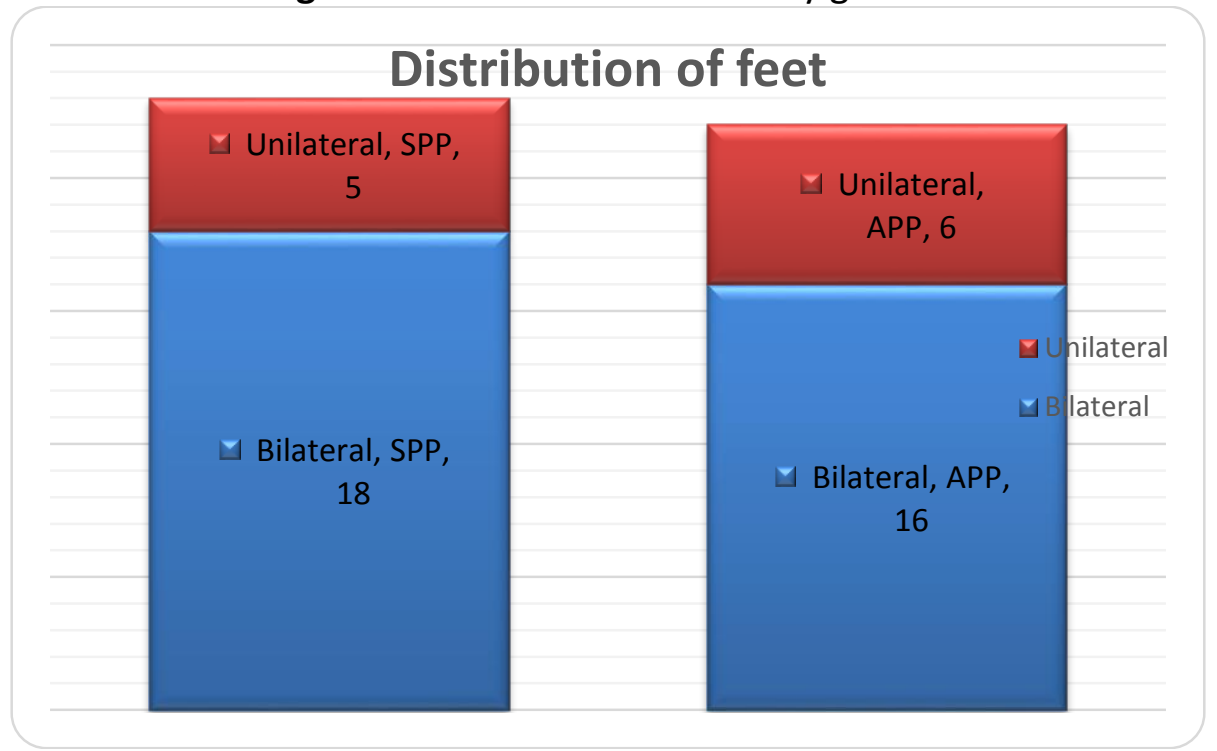

Figure 2. Distribution of feet in both groups 




Figure 3. Number of cast sessions in each group

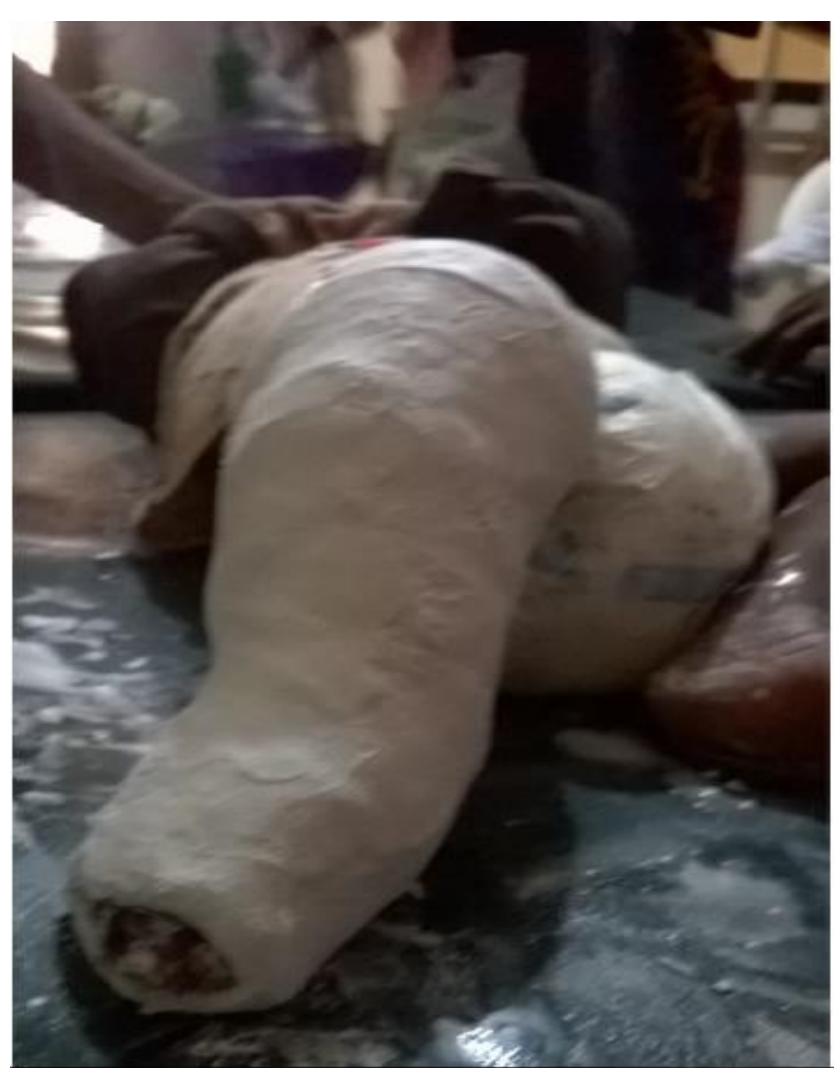

Figure 4. Penultimate cast applied for a patient in the APP group 
All patients had complete correction of their deformity at the end of treatment and all but one had percutaneous Achilles' tenotomy with the last cast kept in place for three weeks to allow the severed Achilles' tendon time to heal. All patients had abduction braces applied on the final day of treatment.

Total treatment duration was found to be significantly shorter in the APP group than in the SPP group ( $p<0.001)$. The APP group completed their treatment within 39 days on average (range 32 - 49 days) compared with an average of 52 days (range $35-77$ days) for the SPP group. The APP group however required a higher number of casts applications compared to the SPP group although this was not statistically significant $(p=0.193)$. The number of casting sessions required in each of the groups is shown in Figure 3.

The patients in the APP group incurred higher treatment costs compared to those in the SPP group. ( $p=0.039$ ). An average of 6650 ( $\$ 33.4$ ) was spent by the patients in the APP group compared to $\$ 5560$ (\$27.9) in the patients in the SPP group.

\section{Discussion}

The male to female ratio amongst the 28 patients recruited was $1.5: 1$. While most studies have shown a male preponderance amongst patients with clubfoot deformity, no evidence was found in the literature suggesting a correlation between the patient's sex and the severity of clubfoot ${ }^{27,28}$. Among the 28 patients studied, the ratio of bilateral to unilateral clubfoot was also 1.5:1. This corresponds well with results from other reports where the ratio of bilateral to unilateral clubfoot varies from $1: 1$ to $2: 1^{27,28}$. Patients with bilateral clubfoot had to have tenotomy and brace application at the same time in both feet. On the rare occasion where one of the feet was ready for tenotomy before the other in bilateral cases, tenotomy was delayed till the second foot was ready according to the Ponseti protocol.

The Ponseti method of clubfoot treatment has attained wide popularity in the last two decades and is currently accepted as the optimal treatment for congenital clubfoot deformity ${ }^{21,22}$. The Ponseti protocol described by Ignatio Ponseti prescribed a rigid sequence of weekly manipulation and cast application. Ponseti believed that a minimum of five days was required between each cast application. This study however employed a shorter duration between casts (twice weekly).

The study showed that patients who were treated with the accelerated Ponseti protocol of twice weekly casts were able to complete their treatment within a shorter period than those who went through the standard Ponseti protocol. The largest comprehensive study of an accelerated Ponseti method was undertaken by Morcuende et al who undertook a retrospective, non-randomized study of 319 feet in 230 patients with clubfoot who were treated over an 11 year period ${ }^{24}$. Their patients were divided into two groups based on their location. One group had an accelerated treatment with five-daily cast changes while the second group had the regular weekly cast changes. Their study showed no difference in the number of casts (four) required for treatment of both groups and consequently a shorter 
overall treatment period in the five-day group compared with the standard weekly casts. Our study showed a shorter treatment duration with the twice weekly accelerated protocol, it however showed a higher number of cast changes to achieve correction with the accelerated protocol compared with the standard protocol. Morcuende et al performed percutaneous tendo Achilles tenotomy for $83 \%$ of their patients ( $85 \%$ of the five day group and $81 \%$ of the seven day group ${ }^{24}$. In our study, all feet except one, had percutaneous Achilles tenotomy (98\%) and she happened to be in the SPP group. The reported tenotomy rates for patients managed by the Ponseti method vary from $<50 \%$ to $100 \%{ }^{24,29}$.

Morcuende et al thought that shorter casting intervals would give rise to problems with swelling in the cast but no patients developed this problem in our study. Harnett et al compared an accelerated Ponseti protocol of three-weekly casts with the standard Ponseti treatment in Malawi ${ }^{25}$. The study involved 61 feet in 40 patients with 29 feet treated with the thrice weekly protocol and 32 feet treated with the standard weekly protocol. Their study showed no difference in the number of casts required between the accelerated group and the standard group. Three of the 19 patients in the accelerated group however did not respond to treatment and had to be switched over to the weekly group. Like this study, theirs did not show any evidence of problems with swelling in the casts despite the accelerated protocol.

Sutcliffe et al reported two cases of a four- weekly casting process using the Ponseti method from Tonga -the country with the highest worldwide incidence of congenital clubfoot ${ }^{8}$. They showed consistent correction of the foot deformity with manipulation and casting, suggesting that manipulation and casting done four times a week is feasible as a treatment alternative for clubfoot. Their cases however involved more manipulation prior to casting than is normally performed. More manipulation prior to cast application for accelerated Ponseti protocols was first advocated by Xu R.J in his study on a twice weekly accelerated Ponseti treatment ${ }^{26}$. Xu's study also showed a shorter casting period in patients with the accelerated group compared with those managed with the regular Ponseti treatment. There was no significant difference in the number of casts required for either group in his report. He however advocated for a longer period of manipulation (two minutes) prior to casting in patients undergoing accelerated treatment protocols. Patients in our study had similar duration of manipulation in both the standard and accelerated groups. This may be responsible for the higher number of casts in the APP group in this study as compared to the Xu's study. No reports could be found formally investigating the effect of more manipulation prior to casting though microscopic evaluation of the ligaments in the foot suggests that maintained loading promotes the lengthening or 'uncrimping' of these ligaments ${ }^{30}$. Further investigations will need to be done to clinically evaluate the amount of manipulation that is necessary to provide maximum benefit before each cast application.

Because the direct costs of treatment depends on the number of times casts are applied as well as the number of materials used for each cast, patients in the APP group had on average, higher direct treatment costs than those in the SPP group from this study. The older average age at presentation in the APP group may also have resulted in more materials used for each patient hence increasing their direct costs. Overall cost of treatment 


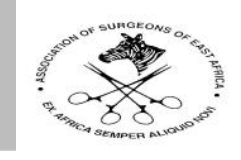

however includes cost of transportation, time off work for the caregiver to attend the clinic as well as cost of relocation to treatment centres for those who live far from the Ponseti clinic. The overall cost benefit analysis will therefore need to take these other indirect costs into consideration.

Patients in locations where skill for percutaneous tenotomy is scarce may benefit from accelerated Ponseti protocols that can enable the manipulation and casting process to be performed early enough to make use of available resources for tenotomy whenever it will be available. This was demonstrated in the report of Sutcliffe et al where a surgeon to perform the tenotomy was not always present ${ }^{8}$.

For the purposes of this study a prolonged follow up of the patients to monitor for recurrence or relapse was not possible. It is therefore not known if there was any difference in the rate of relapse or recurrence between both groups studied. Other studies have failed to show any significant difference in the relapse or recurrence rates between patients managed by the standard Ponseti method and those managed with accelerated methods. The two groups were not matched for age and sex. While there is no evidence to show worse outcomes in any gender, older patients at presentation potentially have longer durations of treatment for the same degree of severity because of possible stiffness of the joints. Randomization was done at presentation and the age of all patients recruited was limited to 90 days of life to reduce this bias

\section{Conclusion}

The study has demonstrated that faster correction of idiopathic clubfoot deformity can be achieved with an accelerated Ponseti protocol of twice weekly casts. The accelerated protocol did not demonstrate any untoward effects in terms of swelling of the feet in the casts. Though the number of casts required to achieve correction was higher with the accelerated protocol, further evaluation will be necessary to show if an increase in direct costs incurred from the casts is offset by the time saved in a faster completion of the treatment process. Further studies will also be necessary to show what socioeconomic circumstances of the patient will enable them benefit more from an accelerated or from the standard Ponseti protocol for management of their clubfeet.

\section{References}

1. Tachdijan M. Paediatric Orthopaedics. 2nd ed. Philadelphia: WB Saunders;1990: 2428.

2. Gupta A, Singh S, Patel P, Patel J, Varshney MK Evaluation of the utility of the Ponseti method of correction of clubfoot deformity in a developing nation. International Orthopaedics2008;32:75-9

3. Anand A, Sala D. Clubfoot: Etiology and treatment. Indian J Orthop2008 42: 22- 28.

4. Ponseti I. Scientific basis of management. In: Staheli I, ed. Clubfoot: Ponseti management. Third ed. Global-HELP, 2009:4.

http://www.global-help.org/Publications/books/(date last accessed 22 November 2014) 
5. Pirani S. Pirani severity scoring. In: Staheli I, ed. Clubfoot: Ponseti management. Third ed. Global-HELP, 2009:27.

http://www.global-help.org/Publications/books/(date last accessed 22 November 2014).

6. Flynn JM, Donohue M, Mackenzie WG. An independent assessment of two clubfootclassification systems. J PediatrOrthop1998; 18:323-7.

7. Noh H, Park SS. Predictive factors for residual equinovarus deformity following Ponseti treatment and percutaneous Achilles tenotomy for idiopathic clubfoot. Acta Orthop2013;84(2):213-7

8. Sutcliffe A, Vaea K, Poulivaati J, Evans AM. 'Fast Casts': Evidence Based and Clinical Considerations for Rapid Ponseti Method. The Foot and Ankle Online Journal 2013; 6(9):2

9. Manzone P. Clubfoot surgical treatment: preliminary results of a prospective comparative study of two techniques. J PediatrOrthop1999; 8:246-50.

10. Zionts LE, Zhao G, Hitchcock K, Maewal J, Ebramzadeh E. Has the rate of extensive surgery to treat idiopathic clubfoot declined in the United States? JBJS 2010 A92: 882-9.

11. Halanski MA, Davison JE, Huang J-C, Walker CG, Walsh SJ, Crawford HA. Ponseti method compared with surgical treatment of clubfoot: a prospective comparison. JBJS 2010; A92:270-278.

12. Laaveg SJ, Ponseti IV. Long term results of treatment of congenital clubfoot. $J$ Bone Joint Surg [Am] 1980;62-A:23-31

13. Herring JA (ed). Congenital talipesequinovarus (clubfoot) in Tachdjian's Paediatric Orthopaedics, vol. 2, 3rd ed. Philadelphia: Saunders 2002:927

14. Kite JH. The treatment of congenital clubfoot. SurgGynaecolObstet1935;61:190-200

15. Scher DM. The Ponseti method of treatment of clubfoot. CurrOpinPediatr2006;18(1):22-8

16. Ponseti IV, Smoley EN. Congenital club foot: the results of treatment. J Bone Joint Surg [Am] 1963; 45-A: 261-344.

17. Cooper DM, Dietz FR. Treatment of idiopathic clubfoot: a thirty-year follow-up note. J Bone Joint Surg [Am] 1995; 77-A: 1477-89.

18. Morcuende JA, Dolan LA, Dietz FR, Ponseti IV. Radical reduction in the rate of extensive corrective surgery for clubfoot using the Ponseti method. Pediatrics 2004; 113:376-80.

19. Abbas M, Qureshi OA, Jeelani LZ, et al. Management of congenital talipesequinovarus by Ponseti method: a clinical study. J Foot Ankle Surg2008; 47:541- 5.

20. Adegbehingbe OO, Oginni LM, Ogundele OJ, et al. Ponseti clubfoot management: changing surgical trends in Nigeria. lowa Orthop J 2010;30:7-14.

21. Steinman S, Richards BS, Faulks S, Kaipus K. A comparison of two nonoperative methods of idiopathic clubfoot correction: the Ponseti method and the French functional (physiotherapy) method. Surgical technique. JBJS.2009; 91A (2):299- 312.

22. Carroll NC. Clubfoot in the twentieth century: where we were and where we may be going in the twenty-first century. J PediatrOrthop. 2012; 21:1-6. 
23. Ponseti IV. Congenital clubfoot -fundamentals of treatment. New York: Oxford University Press;1996:61-81

24. Morcuende J, Abbasi D, Dolan L. Results of an accelerated Ponseti protocol for clubfoot. J Pediatr2005 25: 623-625.

25. Harnett P, Freeman R, Harrison WJ, Brown LC, Beckles V. An accelerated Ponsetiversus the standard Ponseti method. J Bone Joint Surg [Br] 2011;93- B:404-8

26. Xu RJ. A modified Ponseti method for the treatment of idiopathic clubfoot: a preliminary report. J PediatrOrthop2011 31: 317-319.

27. Bowyer G (ed) The ankle and foot In: Apley's system of orthopaedics and fractures, 9th ed. London: Hoder Arnold, 2010:591

28. Alsousou J, Banaskiewicz PA (ed) Paediatric oral topics In: Postgraduate Orthopaedics.1st ed. Cambrige: University Press, 2009:386

29. Kampa R, Binks K, Dunkley M, Coates C. Multidisciplinary management of clubfeet using the Ponseti method in a district general hospital setting. J Child Orthop 2008;2:463-467

30. Ponseti I. Treatment of congenital club foot. JBJS 1992 74: 448-454. 